\title{
DETERMINAÇÃO DO ÍNDICE DE ESTRESSE HÍDRICO EM CULTURA DO FEIJOEIRO COM TERMÔMETRO DE INFRAVERMELHO
}

\author{
Edemo João Fernandes ${ }^{1}$ \\ Departamento de Engenharia Rural, Faculdade de Ciências Agrárias e Veterinárias, Universidade Estadual \\ Paulista, Jaboticabal, SP. ejfernan@fcav.unesp.br
}

\section{RESUMO}

Por intermédio de um termômetro infravermelho portátil foram realizadas leituras diárias de temperaturas da cobertura vegetal e do ar em cultura do feijoeiro submetida a quatro regimes de dotação hídrica, a fim de se calcular o Índice de Estresse Hídrico Diário (IEHD). Com exceção da Testemunha (sem irrigação), todos os outros regimes de aplicação de água não ocasionaram estresse hídrico na cultura. A maior produtividade foi obtida pelo tratamento que teve o menor número de irrigações e, entre os tratamentos que tiveram o mesmo número de irrigações, a maior produtividade foi obtida com o que recebeu maior quantidade de água aplicada. A maior freqüência de aplicação de água não resultou em maior produtividade. As metodologias utilizadas para o planejamento das irrigações foram eficientes para a reposição de água no solo. O índice de estresse hídrico diário (IEH) foi eficaz na indicação do estresse hídrico da cultura, apresentando sempre valores negativos em condições de umidade do solo favorável ao desenvolvimento da cultura.

UNITERMOS: Estresse hídrico, termômetro infravermelho, irrigação, feijão.

\section{FERNANDES, E.J. WATER STRESS INDEX DETERMINATION ON BEAN CROP WITH INFRARED THERMOMETRY}

\section{ABSTRACT}

In order to calculate the daily stress degree of a bean crop submitted to four water regime applications, cover crop and air daily measurements temperatures were accomplished by using a hand-held infrared thermometer. The treatments did not present crop water stress except the control (without irrigation). The highest yield was obtained by the treatment that received less irrigation frequency, and among the treatments that had the same number of irrigation. The largest yield was obtained with the one that received larger amount of applied water. The largest irrigation frequency did not result in larger productivity. The methodologies used for the irrigation planning were efficient for the replacement of soil water. The daily stress degree index was effective in determining crop water stress; and it was reliable presenting negative values in good water soil condition.

\footnotetext{
${ }^{1}$ Professor Adjunto, Departamento de Engenharia Rural, Faculdade de Ciências Agrárias e Veterinárias, UNESP. CEP 14870000, Jaboticabal, SP, (016)3209 2637.
} 
KEYWORDS: Water stress, infrared thermometer, irrigation, bean.

\section{3, INTRODUÇÃO}

O manejo de água em culturas irrigadas é função dos fatores relacionados à cultura, clima e solo, os quais determinarão a quantidade de água a ser aplicada e o momento da irrigação. Diversos métodos para determinação da quantidade de água a ser utilizada e a época de sua aplicação têm sido estudados a fim de racionalizar o uso da água, tendo em vista o aumento da demanda desse produto em vários setores da economia. Em vista disso, métodos que analisam características de plantas têm sido empregados, dentre eles, a determinação da temperatura da cobertura vegetal, a qual tem sido utilizada para estimar índices de estresse hídrico da cultura.

A partir das considerações teóricas de Jackson (1982) e resultados empíricos de Nakayama \& Bucks (1984) e Idso \& Reginato (1982) foi enfatizada a validade e a utilidade do índice de estresse hídrico da planta na monitoração do estresse hídrico e planejamento da irrigação em culturas de climas áridos.

A utilização de índices de estresse hídrico somente tornou-se possível com o advento da termometria infravermelha. A este respeito, Stockle \& Dugas (1992) relataram que o uso de medidas de temperatura da cobertura vegetal, para o planejamento da irrigação, tornou-se prático com o desenvolvimento dos termômetros infravermelhos capazes de medirem a radiação térmica emitida.

Ainda sobre este mesmo assunto, Khera \& Sandhu (1986) relataram que avanços tecnológicos recentes em termômetros infravermelhos portáteis permitem seu uso para medidas rápidas e precisas da temperatura da cobertura vegetal. Esse desenvolvimento tem renovado o interesse de pesquisadores no uso da temperatura da folhagem de plantas como um indicador do estado da água na planta.

Os termômetros infravermelhos medem o fluxo da radiação térmica entre dois objetos. O fluxo líquido de energia é do objeto mais quente para o mais frio e segue a lei de Stefan-Boltzman. A radiação infravermelha tem propriedades óticas da radiação visível, tais como a reflexão e refração. A medida de temperatura da superfície radioativa de um alvo, com termômetro infravermelho, consiste na medida da diferença entre a temperatura do sensor do termômetro e o alvo, chamada de temperatura relativa, e na medida da temperatura do sensor. A temperatura relativa de um alvo é obtida do sensor que capta uma amostra da radiação térmica infravermelha, convertendo-a em um sinal elétrico, cuja voltagem é proporcional a radiação captada. Este valor é convertido para temperatura relativa usando uma relação linear temperatura-voltagem. A temperatura do sensor é medida com um termistor. A temperatura do alvo é a soma da do sensor com a relativa (Wanjura \& Upchurch, 1991).

Quando uma cultura começa a sofrer estresse por falta de água, a condutância dos estômatos e a troca de calor latente são reduzidas, o efeito de resfriamento da evaporação diminui e as folhas apresentam-se com maior temperatura em relação a uma cultura não estressada (Stockle \& Dugas, 1992).

Khera \& Sandhu (1986) relataram que a temperatura média da cobertura vegetal de cultura estressada foi sempre mais alta do que a não estressada e a diferença variou de $0,1{ }^{\circ} \mathrm{C}$ (logo após a irrigação) a $7,7{ }^{\circ} \mathrm{C}$ (antes da irrigação). No geral, culturas estressadas 
apresentavam temperaturas maiores $\left(3\right.$ a $\left.5^{\circ} \mathrm{C}\right)$.

Resultados de estudos que tem usado temperatura da superfície vegetal para controle da irrigação tem sugerido que diferenças menores do que $1{ }^{\circ} \mathrm{C}$ podem produzir diferenças significativas em produção (Wanjura et al., 1989, citados por Wanjura \& Upchurch, 1991).

Um dos problemas para se obter os índices de estresse hídrico esta na manipulação do aparelho, ou seja, o seu correto posicionamento para obtenção da leitura.

Wanjura \& Upchurch (1991) informaram que a superfície de uma cobertura vegetal é muito complexa, consistindo de superfícies de folhas iluminadas e sombreadas com orientações variando segundo o ângulo de visada do termômetro infravermelho. A temperatura da cobertura vegetal é uma média influenciada pelos componentes radioativos da área abrangida pelo ângulo de visada.

Um estudo de Hatfield et al. (1984) demonstrou que a composição da estrutura vegetal afeta a temperatura aparente medida pelo termômetro infravermelho. Temperaturas da cobertura vegetal de trigo com panículas, medidas a um ângulo horizontal de $30^{\circ}$ foram $2^{\circ} \mathrm{C}$ mais alto do que sem panículas. A diferença da temperatura aparente foi atribuída às panículas, que correspondiam a 40\% da área visada, não sendo órgão ativo da transpiração.

Temperaturas da cultura de cevada medidas com termômetro infravermelho, utilizando ângulo de visada nadir e $30^{\circ}$ da horizontal, indicou que as temperaturas obtidas da composição planta-solo nu pelo ângulo nadir foi 0,5 a $11,5{ }^{\circ} \mathrm{C}$ mais alta do que a determinada pelo ângulo de visada de $30^{\circ}$ (Heilman et al., 1981).

A influência do ângulo de visada e cobertura do solo sobre a temperatura da cobertura vegetal de trigo foi minimizada pelo posicionamento do termômetro infravermelho a $35^{\circ}$ da horizontal em relação ao raio solar (Huband \& Monteith, 1986).

Macguire et al. (1989), após revisão de vários trabalhos recentes sobre o uso do termômetro infravermelho, relataram que o ângulo de visada, a estrutura da vegetação e porcentagem de solo coberto podem afetar as medidas do sensor térmico.

Medidas da temperatura da cobertura vegetal são altamente sensíveis ao ângulo de visada do aparelho e sua relação com o ângulo zenital solar (Huband \& Monteith, 1986; Fuchs, 1990). Entretanto, a padronização e consistência de procedimentos são importantes.

Algumas insuficiências desta técnica de planejamento da irrigação incluem a dificuldade em medir a temperatura da cobertura vegetal em estádios iniciais do desenvolvimento da cultura (Jackson et al., 1981; Howell et al., 1984) e o fato de que este processo não indica a quantidade de água a aplicar (Nielsen, 1990).

\section{MATERIAL E MÉTODOS}

O ensaio foi instalado na Área Demonstrativa e Experimental de Irrigação - ADEI, da FCAVJ/UNESP, Câmpus de Jaboticabal -SP. As coordenadas geográficas são $21^{\circ} 15^{\prime} 22^{\prime}$ ', de Latitude Sul e 48 $18^{\prime} 58^{\prime}$ " de Longitude Oeste, sendo a altitude média de $570 \mathrm{~m}$, 
apresentando clima tipo Cwa (subtropical) de acordo com a classificação de Köppem, com 22 ${ }^{\circ} \mathrm{C}$ de temperatura média anual, $70 \%$ de umidade relativa média do ar e $1400 \mathrm{~mm}$ de precipitação média anual.

O solo da área experimental é classificado como Latossolo Vermelho eutroférrico argiloso, horizonte A moderado, textura argilosa, relevo suave ondulado.

O preparo do solo da área experimental foi o convencional, constando de arações, gradagens e subsolagem. A adubação da cultura foi $300 \mathrm{~kg} \mathrm{ha}^{-1}$ dos elementos N-P-K na formulação 2-20-20 de acordo com recomendação baseada na análise do solo. A área total do experimento constou de 1,0 ha sendo a área útil de cada parcela de 0,2 ha.

Utilizou-se o sistema de irrigação por aspersão convencional com espaçamento de 18 X 18 m, sendo instalada na área experimental a cultura do feijoeiro (Phaseolus vulgaris L.), cultivar Carioca-80, com espaçamento de $0,50 \mathrm{~m}$ entre linhas. A emergência de $70 \%$ das plântulas ocorreu em 12/05/95.

Os tratamentos foram designados como: T1 - Irrigação baseada em estimativa da evapotranspiração da cultura (ETc); Tensiometria (T2 e T3) - Irrigação baseada em dados fornecidos pela leitura de tensiômetros e Testemunha (T4) - irrigada somente para promover a emergência das plântulas, provendo o solo com a mesma condição hídrica dos outros tratamentos e manutenção inicial da cultura.

Em todas as parcelas foram instaladas três baterias de tensiômetros, nas profundidades de 0,$20 ; 0,40 ; 0,60 ; 0,80$ e 1,00 m. A metodologia adotada, para o início da irrigação nas parcelas dos tratamentos T2 e T3, constou em marcar a tensão de água no solo a $0,40 \mathrm{~m}$ de profundidade, quando a média das leituras do tensiômetro à $0,20 \mathrm{~m}$ atingisse $40 \mathrm{kPa} \mathrm{e}$ $60 \mathrm{kPa}$, respectivamente (Katerji \& Hallaire, 1984). O valor da tensão da água no solo a $0,40 \mathrm{~m}$ que iniciou a irrigação do tratamento T2 foi de $21,1 \mathrm{kPa}$ e o do T3 $24,8 \mathrm{kPa}$. A partir desses momentos, as irrigações nas parcelas desses tratamentos foram sempre realizadas quando as tensões médias na profundidade de $0,40 \mathrm{~m}$ atingissem os valores citados. A quantidade de água aplicada foi função da somatória da evapotranspiração da cultura (ETc) no intervalo entre duas irrigações consecutivas. matemática:

Para o cálculo do potencial da água no solo foi utilizada a seguinte expressão

$$
\Psi_{\mathrm{m}}=\left(-12,6 \mathrm{~h}_{1}+\mathrm{h}_{2}\right) 9,810^{-2}
$$

na qual $\Psi_{\mathrm{m}}$ é o potencial matricial de água no solo $(\mathrm{kPa}), \mathrm{h}_{1}$ a altura da coluna de mercúrio $(\mathrm{cm})$, $\mathrm{h}_{2}$ a distância do nível de mercúrio no reservatório ao centro da cápsula de cerâmica $(\mathrm{cm})$.

Em cada profundidade foi determinado o potencial de água no solo, pelo método da câmara de pressão de Richards (Richards, 1941), até -100 kPa, sendo a capacidade de campo determinada por meio da curva de retenção de água no solo, ajustada pela expressão matemática desenvolvida por Genuchten (1980):

$$
\theta=\theta_{r}+\frac{\left(\theta_{s}-\theta_{r}\right)}{\left[1+(\Psi \alpha)^{m}\right]^{n}}
$$

em que. $\theta$ é a umidade do solo a base de volume $\left(\mathrm{cm}^{3} \mathrm{~cm}^{-3}\right), \Psi$ o potencial de água no solo $(\mathrm{cm}$ c. a), $\theta$ r a umidade residual $\left(\mathrm{cm}^{3} \mathrm{~cm}^{-3}\right), \theta$ s a umidade de saturação $\left(\mathrm{cm}^{3} \mathrm{~cm}^{-3}\right)$ e $\alpha, \mathrm{m}$ e $\mathrm{n}$ os parâmetros empíricos do modelo matemático.

A reserva utilizável de água no solo (RU, em mm) e a água disponível (AD) foram determinadas por meio das seguintes equações:

$$
\mathrm{RU}=(\theta \mathrm{cc}-\theta \mathrm{pmp}) \mathrm{z}
$$




$$
\mathrm{AD}=(\theta \mathrm{cc}-\theta \mathrm{a}) \mathrm{z}
$$

em que $\theta \mathrm{cc}, \theta$ pmp e $\theta$ a são as umidades volumétricas na capacidade de campo, ponto de murcha permanente e atual do solo $\left(\mathrm{cm}^{3} \mathrm{~cm}^{-3}\right)$, respectivamente, e $\mathrm{z}$ a profundidade efetiva das raízes $(\mathrm{mm})$.

A irrigação das parcelas do tratamento $\mathrm{T} 1$ foi efetuada por intermédio de valores estimados da ETc, obtidos por meio de dados fornecidos por um tanque classe A e do coeficiente da cultura (Doorenbos \& Kassan, 1979), sendo a ETc calculada por intermédio das seguintes equações:

$$
\begin{aligned}
& \mathrm{ETo}=\text { Eca }_{\mathrm{p}} \\
& \mathrm{ETc}={\text { ETo } \mathrm{K}_{\mathrm{c}}}^{\mathrm{E}}
\end{aligned}
$$

em que ETo é a evapotranspiração de referência $(\mathrm{mm})$, Eca a evaporação medida no tanque classe A (mm), Kp o coeficiente do tanque classe A, ETc a evapotranspiração da cultura (mm) e Kc o coeficiente da cultura.

A parcela do tratamento T4 foi irrigada após a semeadura para favorecer a emergência das plântulas, aos 20 dias (31/5) após a emergência das plântulas (d.a.e.), em 28 d.a.e. (08/6) e, após essa data, nenhum aporte de água foi efetuado, até o final do ciclo da cultura, ocorrendo apenas precipitações pluviométricas naturais. Os demais tratamentos também receberam as mesmas lâminas de água aplicadas no T4 (31,4 mm) nas duas datas.

A determinação do IEHD (Índice de Estresse Hídrico Diário) foi efetuada em função das medidas da temperatura da cobertura vegetal e do ar ambiente, ao mesmo tempo, com um termômetro infravermelho portátil de ângulo de visada (ângulo sólido) de $3^{\circ}$, sendo utilizada uma inclinação do aparelho de aproximadamente $30^{\circ}$ com a horizontal, para a visada. Foram efetuadas 10 visadas em cada parcela, realizadas próximas ao meio dia solar. $\mathrm{O}$ valor da temperatura da cobertura vegetal e do ar, em cada parcela, foi obtido pela média das 10 visadas.

Para o cálculo do IEHD utilizou-se a seguinte equação (Idso et al., 1977 e Jackson et al., 1977):

$$
\mathrm{IEHD}=(\mathrm{Tmc}-\mathrm{Tma})
$$

em que Tmc é a temperatura média da cobertura vegetal $\left({ }^{\circ} \mathrm{C}\right)$, Tma a temperatura média do ar $\left({ }^{\circ} \mathrm{C}\right)$,

A produção dos grãos foi avaliada, colhendo-se as vagens do feijoeiro em uma área útil de $300 \mathrm{~m}^{2}$ de cada parcela, reservada especialmente para esta operação e determinando-se o peso dos grãos com $13 \%$ de umidade.

\section{RESULTADOS E DISCUSSÃO}

A Tabela 1 apresenta as quantidades médias de lâminas de água aplicadas por irrigação para cada tratamento e as precipitação, com suas respectivas datas de ocorrência. A quantidade total de água recebida das chuvas ocorridas durante o ciclo da cultura foi de 53,6 $\mathrm{mm}$. Nota-se que o tratamento T1 apresentou o maior número de irrigações (oito) bem como maior quantidade de água aplicada por irrigação, enquanto os tratamentos T2 e T3 apresentaram o mesmo número de irrigações efetuadas (cinco), diferindo apenas na quantidade recebida.

O tratamento T2 recebeu $77,7 \%$ da lâmina total de água aplicada no T1, enquanto que o T3 recebeu 87,3\%. Essa porcentagem entre os tratamentos T2 e T3, foi de 89,1\%. Durante o transcorrer do experimento ocorreram quatro precipitações perfazendo um total de 
$27,8 \mathrm{~mm}$, o que possibilitou o desenvolvimento da cultura no tratamento T4.

Tabela 1. Lâminas média de água aplicadas em cada tratamento e precipitações, em mm, ocorridas durante o desenvolvimento do experimento.

\begin{tabular}{|l|l|l|l|l|l|}
\hline Data & T1 $(\mathrm{mm})$ & T2 $(\mathrm{mm})$ & T3 $(\mathrm{mm})$ & Data & $\begin{array}{l}\text { Chuvas } \\
(\mathrm{mm})\end{array}$ \\
\hline $31 / 5$ & 31,4 & 31,4 & 31,4 & $18 / 6$ & 7,8 \\
\hline $8 / 6$ & 31,4 & 31,4 & 31,4 & $25 / 6$ & 4,6 \\
\hline $20 / 6$ & 29,4 & & & $9 / 7$ & 7,4 \\
\hline $27 / 6$ & & 42,5 & & $10 / 7$ & 8,0 \\
\hline $30 / 6$ & & & 46,8 & & \\
\hline $7 / 7$ & 34,8 & & & & \\
\hline $12 / 7$ & & 39,6 & & & \\
\hline $17 / 7$ & 45,8 & & & & \\
\hline $21 / 7$ & & & 83,8 & & \\
\hline $27 / 7$ & 33,0 & 61,8 & & & \\
\hline $3 / 8$ & 24,9 & & 38,7 & & \\
\hline $8 / 8$ & 35,3 & & & & \\
\hline & & & & & \\
\hline Total & 266,0 & 206,7 & 232,1 & & \\
\hline
\end{tabular}

A reserva utilizável de água no solo na camada de 0 a $0,60 \mathrm{~m}$ é de $46,3 \mathrm{~mm}$. $\mathrm{Na}$ Figura 1 encontram-se os valores de água disponível no solo na camada de 0 a $0.60 \mathrm{~m}$ para todos os tratamentos, verificando-se que os menores valores foram detectados no T2, dos 95 aos 102 d.a.e.

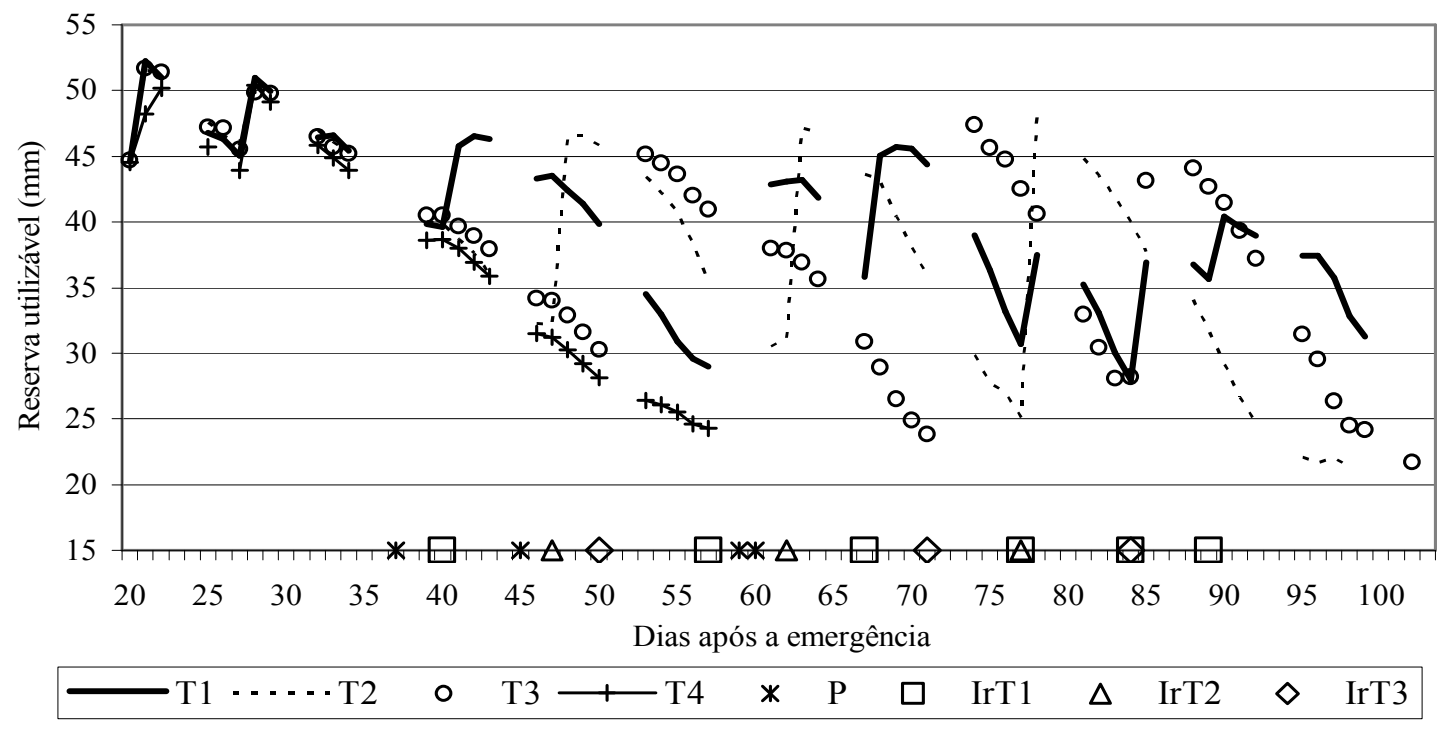

Figura 1. Valores médios de água disponível (AD) no solo até $0.60 \mathrm{~m}$ de profundidade, para todos os tratamentos, chuvas ocorridas (P) e irrigações efetuadas (Ir).

O menor valor encontrado foi $20,9 \mathrm{~mm}$ o que corresponde à $45,1 \%$ da RU. Para T1 o menor valor foi 28,0 registrado aos 84 d.a.e., correspondendo a $60,5 \%$ da RU, enquanto para T3 o valor foi $23,8 \mathrm{~mm}$ ocorrido aos 71 d.a.e. (51,4\% da RIU).

No tratamento T4 o menor valor foi verificado aos 57 d.a.e. $(24,3 \mathrm{~mm})$, porque a 
partir dessa data os tensiômetros instalados na profundidade de $0,20 \mathrm{~m}$ pararam de funcionar. Observa-se que o único tratamento que apresentou um esgotamento de água superior a $50 \%$ da RU (Allen et al, 1998) foi o T2, mas isso ocorreu no estádio de maturação dos grãos.

As duas primeiras irrigações efetuadas nas parcelas de todos os tratamentos foram efetivas para elevar a umidade do solo à capacidade de campo. Em T1, as três últimas irrigações não conseguiram suprir o solo em seu déficit de umidade natural. Em T2 e T3 todas as lâminas de água aplicadas, via irrigações, foram suficientes para atingir ou manter-se próximo à capacidade de campo do solo.

A Figura 2 apresenta os dados médios de IEHD, para todos os tratamentos, verificando-se que dos 45 aos 57 d.a.e. todos os tratamentos apresentaram alguns valores de IEHD positivos no período compreendido entre as irrigações, sendo que o T4 apresentou o maior valor $\left(3,6{ }^{\circ} \mathrm{C}\right)$. De uma maneira geral, os tratamentos, com exceção da testemunha, apresentaram tendências semelhantes durante todo o ciclo de observações. A partir do 58 d.a.e. os tratamentos, à exceção da testemunha (T4), apresentaram valores negativos até aos 87 d.a.e., indicando, que neste período, a cultura não apresentou estresse hídrico, pois a temperatura da cobertura vegetal estava abaixo da temperatura do ar, caracterizando o processo de transpiração da planta, tendo em vista seu conforto hídrico e, consequentemente, diminuição de sua temperatura.

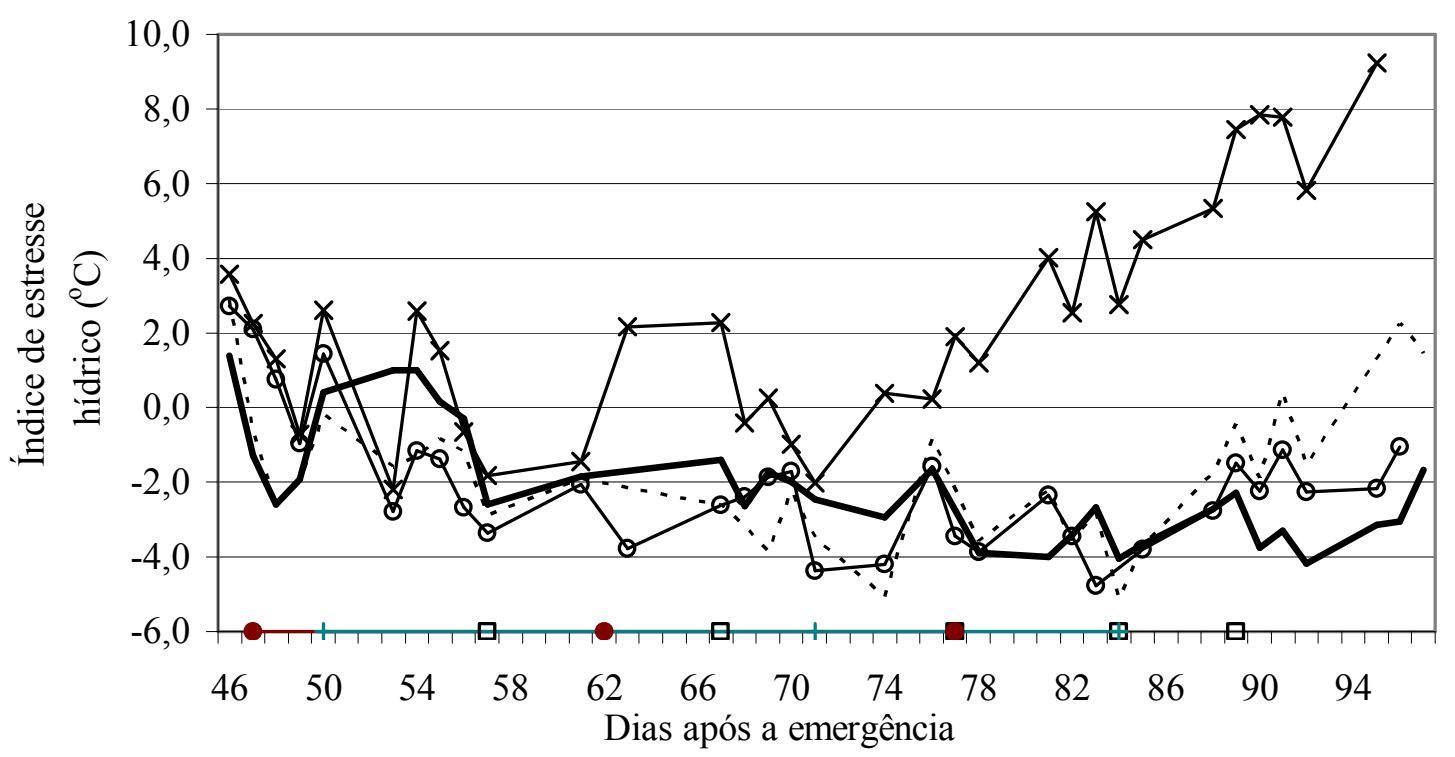

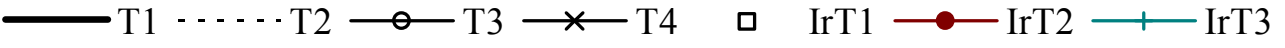

Figura 2. Valores médios do Índice de Estresse Diário, em ${ }^{\circ} \mathrm{C}$, para todos os tratamentos.

Esses resultados estão em conformidade com os dados de umidade do solo, pois, nesse período, o esgotamento de água no solo dos tratamentos não ultrapassou $50 \%$ da RU. O maior valor de IEHD foi obtido pelo tratamento T4 aos 98 d.a.e., atingindo $9,2{ }^{\circ} \mathrm{C}$. A cultura estressada (T4.) apresentou, durante todo o período de medidas, valores de IEHD superiores aos dos outros tratamentos, concordando com os dados obtidos por Khera \& Sandhu (1986). Observa-se, ainda, que toda irrigação fez com que o valores de IEHD diminuíssem, o mesmo acontecendo com as precipitações, como se pode verificar pelo comportamento das plantas do tratamento T4. 
A Figura 3 apresenta os dados de produção média dos grãos em $\mathrm{kg} \mathrm{ha}^{-1}$, observando-se que o tratamento T3 foi o que apresentou maior produtividade, enquanto o T4 foi o que obteve o menor rendimento. O tratamento $\mathrm{T} 1$ que consumiu a maior quantidade de água e teve a maior freqüência de irrigações não foi o mais produtivo, resultado inverso do que ocorreu com a cultura da soja, de acordo com Fernandes (1996). O tratamento T3 teve o mesmo número de irrigações do que o tratamento T2, porém recebeu $25,4 \mathrm{~mm}$ de lâmina de água a mais do que o T1. Portanto, a maior freqüência de irrigação não ocasionou maior produtividade, enquanto que, com a mesma freqüência, o maior rendimento foi do tratamento que recebeu maior quantidade de água.

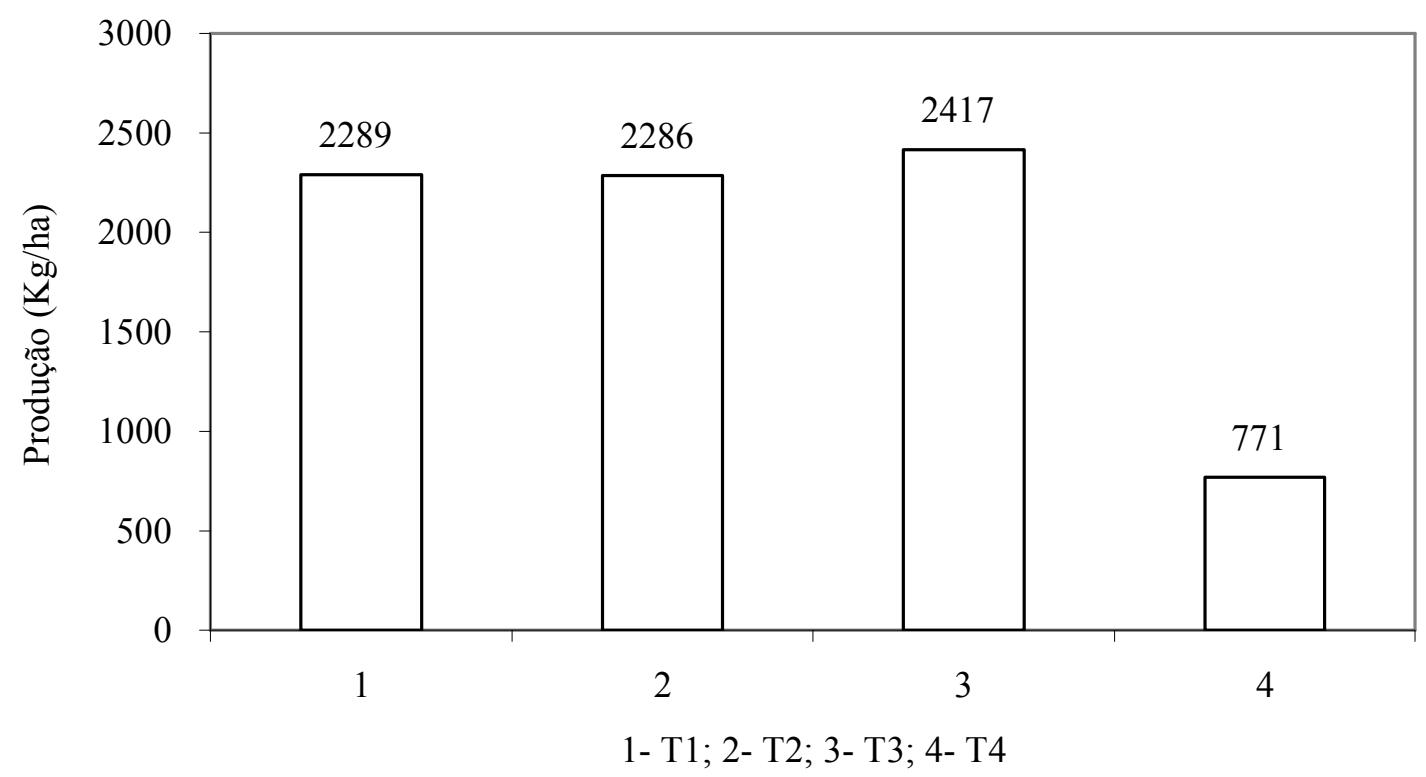

Figura 3. Produtividade de grãos, em $\mathrm{kg} \mathrm{ha}^{-1}$.

\section{CONCLUSÕES}

A cultura do feijoeiro apresentou maior produção quando a aplicação de água por irrigação foi menos freqüente. Em condições idênticas de freqüência de irrigação, a produção foi maior no tratamento que recebeu maior quantidade de água. Os três regimes de irrigação foram eficazes para a cultura do feijoeiro, produzindo IEHD negativo durante os estádios de florescimento e enchimento de grãos. A cultura estressada apresentou sempre maiores valores de IEHD do que as não estressadas.

\section{REFERÊNCIAS BIBLIOGRÁFICAS}

DOORENBOS, J.; KASSAM, A.H. Yield response to water. FAO Irrigation and Drainage, Paper n. 33, Rome, 1979.

FERNANDES, E.J.; BAVARESCO, R.S. Uso do termômetro de infravermelho na 
determinação do índice de estresse hídrico em cultura de soja. Engenharia Agrícola, Jaboticabal, v. 16, n. 1, p. 76-83, 1996.

FUCHS, M. Infrared measurement of canopy temperature and detection of plant water stress. Theory Applied Climatology, v. 42, p. 254-261, 1990.

HATFIELD, J.L.; PINTER, P.J.; CHASSERAY, E.; EZRA, C.E.; REGINATO, R.J.; IDSO, S.B.; JACKSON, R.D. Effects of panicles on infrared thermometer measurements of canopy temperature in wheat. Agricultural and Forest Meteorology, v. 32, p. 7-105, 1984.

HEILMAN, J.L.; HEILMAN, W.E.; MOORE, D.G. Remote sensing of canopy temperature at incomplete cover. Agronomy Journal, Madison, v.73, p. 403-406, 1981.

HOWELL, T.A., MEEK, D.W., PHENE, C.J., DAVIS, K.R., McCORMICK, R.L. Automated weather data collection for research on irrigation scheduling. Transactions of the ASAE, v. 27, n. 2, p. 386-391, 396, 1984.

HUBAND, N.D.S.; MONTEITH, J.L. Radiative surface temperature and energy balance of a wheat canopy. I. Comparison of radiative and aerodynamic canopy temperature. BoundaryLayer Meteorology, v. 36, p. 1-17, 1986.

IDSO, S.B.; JACKSON, R.D.; REGINATO, R.J. Remote sensing of crop yields. Science, Washington, v. 19, n. 6, p. 19-25, 1977.

IDSO, S.B.; REGINATO, R.J. Soil and atmosphere-induced plant water stress in cotton as inferred from foliage temperatures. Water Resource Research, v. 8, p. 143-1148, 1982.

JACKSON, R.D. Canopy temperature and crop water stress. In: HILLEL, D. Advances in irrigation, v. 1, p. 43-80, 1982,.

JACKSON, R.D., IDSO, S.B., REGINATO, R.J., PINTER, P.J. Canopy temperature as a crop water stress indicator. Water Resource Research., v. 17, n. 4, p. 1133-1138, 1981.

JACKSON, R.D., REGINATO, R.J., IDSO, S.B. Wheat canopy temperature: A practical tool for evaluating water requirements. Water Resource Research, v. 13, n. 3, p. 651-656, 1977.

KATERJI, N., HALLAIRE, M. Les grandeurs de reférence utilisables dans l'étude de l'alimentation en eau des cultures. Agronomie, Paris, v. 4, n. 10, p. 999-1008, 1984.

KHERA, K.L.; SANDHU, B.S. Canopy temperature of sugarcane as influenced by irrigation regime. Agricultural and Forest Meteorology, v. 37, p. 245-258, 1986.

McGUIRE, M.J.; BALICK, L.K.; SMITH, J.A.; HUTCHISON, B.A. Modeling directional thermal radiance from forest canopy. Remote Sensing Environmental, v. 27, p. 169-186, 1989.

NAKAYAMA, F.S.; BUCKS, D.A. Crop water stress index, soil water and rubber yield relations for the Guayule plant. Agronomy Journal, v. 76, p. 791-794, 1984. 
NIELSEN, D.C. Scheduling irrigations for soybean with the crop water stress index (CWSI). Field Crops Research, v. 23, p. 103-116, 1990.

STOCKLE, C.O.; DUGAS, W.A. Evaluating canopy temperature-based indices for irrigation scheduling. Irrigation Science, v. 13, p. 31-37, 1992.

WANJURA, D.F.; UPCHURCH, D.R. Infrared thermometer calibration and viewing method effects on canopy temperature measurement. Agricultural and Forest Meteorology, v. 55, p. 309-321, 1991. 